\title{
Utilização do QFD no desenvolvimento de novos produtos: uma análise das publicações em periódicos nacionais
}

\author{
Maurício Johnny Loos \\ Universidade Federal de Santa Catarina - UFSC \\ mauricioloos@hotmail.com \\ Paulo Augusto Cauchick Miguel \\ Universidade Federal de Santa Catarina-UFSC \\ cauchick@deps.ufsc.br
}

\section{RESUMO}

Com a exigência cada vez maior dos clientes, as organizações necessitam encontrar alternativas de diversificar seus produtos e desenvolvê-los com agilidade, visando aumentar sua participação no mercado. Diante disto, o Quality Function Deployment (QFD) ou Desdobramento da Função Qualidade mostra-se como uma alternativa importante para traduzir em requisitos de qualidade as demandas exigidas pelos clientes. Nesse sentido, o presente trabalho tem o objetivo de realizar uma análise bibliográfica de publicações sobre o QFD em periódicos nacionais, especificamente relacionada ao desenvolvimento de novos produtos. O trabalho caracteriza-se como teórico conceitual, uma vez que busca analisar a literatura, por meio da busca de artigos em bases de dados de periódicos nacionais, seguida pela organização e análise dessas publicações. Os resultados possibilitaram uma estratificação sobre o tema de acordo com os periódicos específicos, setores industriais e tipos de estudos, predominando o setor voltado para a indústria manufatureira e a utilização do estudo de caso como abordagem metodológica de pesquisa. Os resultados apontam que, nas publicações sobre QFD no Brasil, o tema “desenvolvimento de novos produtos” é o que apresenta a maior quantidade de publicações (42\%), dentre um total de quinze temas identificados.

Palavras-chave: QFD. Desdobramento da Função Qualidade. Desenvolvimento de Novos Produtos. Revisão da Literatura.

\begin{abstract}
With the growing requirement of customers, organizations need to find alternatives to diversify their products and develop them quickly, aiming to increase their market share. In this sense, Quality Function Deployment (QFD) shows up as an important alternative to translate quality requirements to the demands required by the customers. In this context, this paper aims at carrying out a literature analysis on QFD in Brazilian journals, specifically related to the development of new products. The work is characterized as a theoretical, as it seeks to analyze the literature through the search for articles in the national databases. It also shows the organization and analysis of those publications. The results allowed stratification on the subject according to the specific journals, industry sectors and types of studies. The predominant sector is the manufacturing industry and the use of case study is the most adopted methodological research approach. The results identified that, within the publications on QFD in Brazil, the subject is 'developing new products’ which has the highest quantity of publications (42\%) among a total of fifteen identified subjects.
\end{abstract}

Keywords: QFD. Quality Function Deployment. New Product Development. Literature Review. 


\section{Introdução}

As decisões que envolvem o desenvolvimento de novos produtos consideram uma multiplicidade de conceitos, métodos e ferramentas. Dentre os existentes, o Quality Function Deployment (QFD) ou Desdobramento da Função Qualidade é apontado como um dos métodos disponíveis para auxiliarem nestas decisões (BURKE et al., 2002). O uso do QFD permite a obtenção de índices de priorização que incorporam tanto requisitos de mercado quanto requisitos técnicos. Os requisitos de mercado englobam a qualidade demandada pelos clientes, enquanto que os requisitos técnicos referem-se à estrutura do produto e do processo (ANZANELLO et al., 2009).

Diante de um cenário com as demandas referentes à qualidade e performance dos produtos, sua diversificação, e busca de maior precisão nos atributos dos produtos, o QFD desempenha um papel fundamental para o desenvolvimento de novos produtos. Nesse contexto, busca-se realizar uma análise das publicações associadas ao tema, particularmente na engenharia de produção. Assim, o presente trabalho tem como propósito principal responder a seguinte questão de pesquisa: quais são as características de publicações em periódicos (nacionais) sobre QFD envolvendo desenvolvimento de novos produtos no país? Por meio de uma busca e análise bibliográfica sobre o tema QFD combinado com o tema desenvolvimento de novos produtos, o presente trabalho busca então identificar um quadro geral sobre o tema em periódicos no Brasil, mostrando os vários assuntos encontrados nos artigos sobre QFD, os setores que utilizam o QFD para o desenvolvimento de novos produtos, bem como os periódicos que possuem publicações no tema. Todas as fontes de pesquisa utilizadas são nacionais, sendo intencional o recorte nas práticas divulgadas especificamente no país, possibilitando-se a expansão da análise futuramente. Para cumprir seus objetivos, o trabalho primeiramente estabelece o referencial teórico sobre o QFD, seguido pelos métodos e técnicas adotados, resultados da análise das publicações e, finalmente, suas conclusões.

\section{Base teórica sobre o QFD}

O QFD é um dos métodos que as empresas têm buscado introduzir para dar suporte ao desenvolvimento de novos produtos. O método tem por objetivo integrar as necessidades dos clientes em todo o ciclo de desenvolvimento de um novo produto. O QFD converte as exigências dos usuários em características da qualidade (especificações) e as transfere para as etapas subsequentes de desenvolvimento de produto, por meio de desdobramentos sucessivos (FORMAGGIO; CAUCHICK MIGUEL, 2009). Segundo King (1987), o QFD é uma ferramenta multifuncional que permite às organizações priorizarem as demandas dos consumidores e, em função disso, desenvolverem respostas inovadoras para as necessidades dos clientes, que sejam efetivas em termos de custo e qualidade. O mesmo autor supracitado afirma que o QFD pode direcionar uma implementação bem sucedida de um novo produto ou processo, na medida em que envolve todos os departamentos: marketing, pesquisa e desenvolvimento, produção, controle de qualidade, vendas e serviços, etc.

O QFD é uma abordagem permite que as empresas se tornem pró-ativas para os problemas de qualidade, em vez de tomar uma posição reativa, e que ajam sobre as reclamações dos clientes (BÜYÜKÖZKAN; ÇIFÇI, 2013). Ainda segundo os autores citados, as aplicações bem sucedidas de QFD basicamente, dependem de uma comunicação eficaz entre os membros da equipe para chegar a um consenso e atribuir níveis de importância que refletem as preferências de cada membro individual.

OQFD éum método (RIBEIRO et al., 2001; BOUCHEREAU; ROWLANDS, 2000):

(i) de gestão, auxiliando no gerenciamento de projetos;

(ii) de planejamento onde os esforços de engenharia são transferidos para a fase de desenvolvimento;

(iii) para a solução de problemas que aponta o quê precisa ser feito e como pode ser feito;

(iv) que facilita a modelagem do conhecimento;

(v) que facilita a documentação de informações;

(vi) que facilita o transporte de informações; e

(vii) que, por discussões multisetoriais de engenharia simultânea, permite maior criatividade.

Cauchick Miguel e Carnevalli (2006) colocam que o QFD é um método eficaz na identificação e tradução das necessidades e expectativas dos clientes em relação aos atributos de um produto ou serviço. O método QFD enfatiza os itens importantes, tanto do ponto de vista dos clientes quanto da empresa, sendo um mecanismo que possibilita alcançar vantagens competitivas no desenvolvimento de produtos (CAUCHICK MIGUEL et al., 2003). Para atingir a satisfação dos clientes, o QFD certifica-se de que todas as atividades e operações da empresa são dirigidas pela voz do cliente (NAHM, 2013).

Estruturalmente, o QFD é constituído de uma série de matrizes inter-relacionadas, fazendo com que a informação gerada por uma matriz, em geral, constitua-se na entrada da matriz subseqüente ou das matrizes posteriores (ANZANELLO et al., 2009). Os quatro ciclos QFD propostos são (FERNANDES; REBELATO, 2006): 
1) desenvolvimento das necessidades dos clientes: transforma as expectativas verbalizadas pelos clientes em requisitos que o sistema deve conter, ou seja, traduz os desejos expressos em “necessidades” exigidas para o "sistema produto";

2) projeto do produto e componentes: transforma as necessidades exigidas para o sistema em requisitos do produto e dos componentes;

3) projeto do processo: transforma as características do produto e dos componentes em requisitos do processo, ou seja, estabelece os processos necessários e seus parâmetros; e

4) projeto do controle do processo: transforma as características de cada processo em requisitos de controle, ou seja, o método estabelece os meios, mecanismos e métodos para o controle dos processos.

Na sua forma tradicional, o QFD pode ser apresentado contemplando quatro matrizes formadas por: (i) matriz da qualidade; (ii) matriz do produto (em referência a bens de consumo); (iii) matriz de processos; e (iv) matriz de recursos. Assim, o uso do QFD fornece o suporte para as equipes de desenvolvimento de produtos. Isto se deve ao fato de que essas quatro matrizes relacionam a demanda do consumidor com as especificações de engenharia, possibilitando o projeto dos componentes do produto e a definição das especificações das variáveis dos processos de produção (CHENG; MELO FILHO, 2007; GOVERS, 1996; AKAO, 1990). A Figura 1 ilustra um modelo de aplicação do método QFD.

Figura 1 - Modelo de aplicação do método QFD.

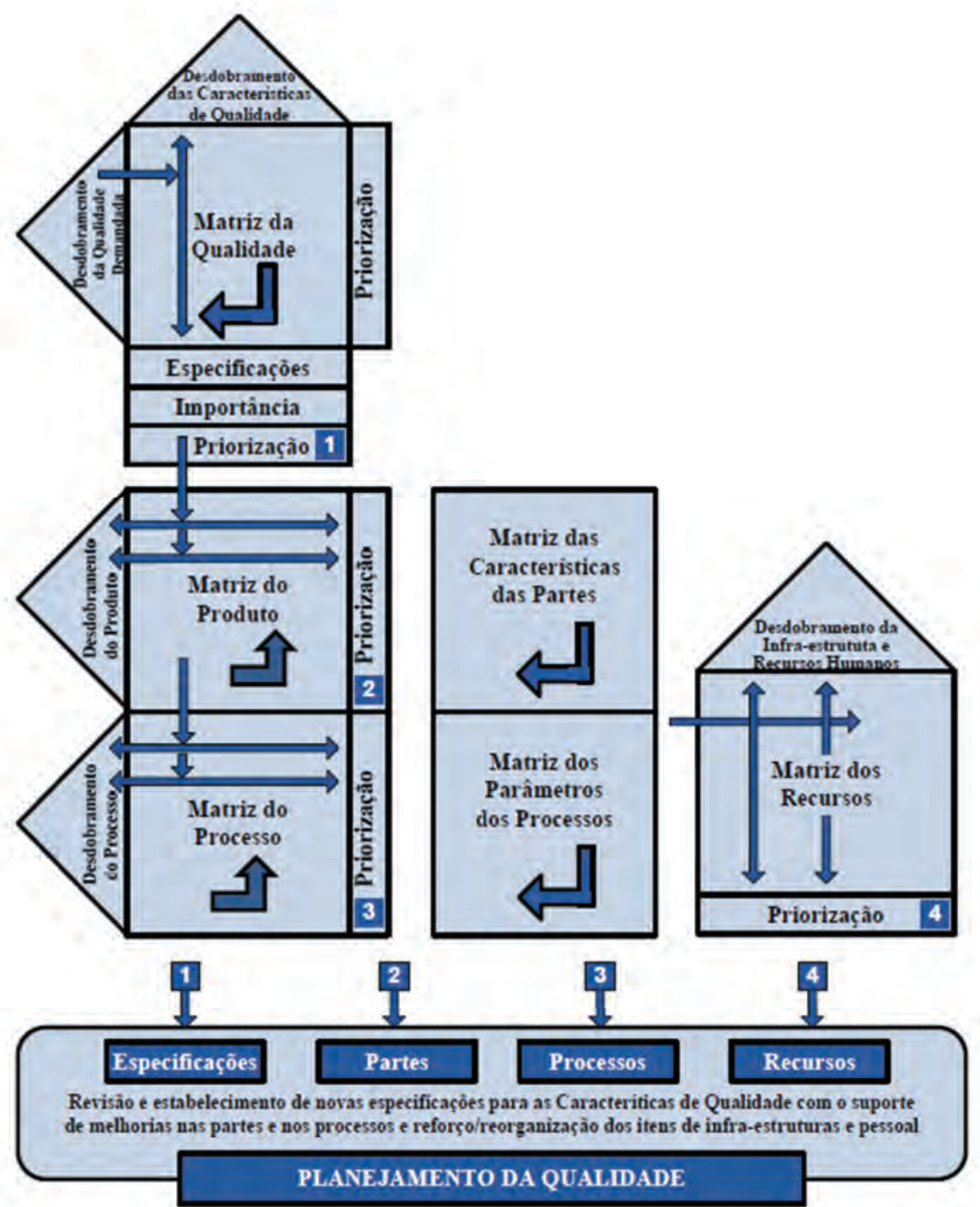

Fonte: Ribeiro et al. (2001). 
A primeira matriz do QFD, que é a matriz da qualidade, é formada pela união da "tabela de desdobramento da qualidade exigida” com a "tabela de desdobramento das características da qualidade” (AKAO, 1996). Nessa matriz são realizadas relações de três tipos (CHENG et al., 1995): extração (qualitativa), conversão (quantitativa) e de correlação (intensidade). Quando os elementos de uma tabela são obtidos a partir de outra acontece a extração; a correlação consiste em identificar o grau de influência (forte, média, fraca e inexistente) que um item de uma tabela exerce sobre outra; e a conversão consiste em transferir a importância, ou seja, o peso relativo a cada item de uma tabela para os itens de outra tabela, através das correlações identificadas no interior da matriz.

Na matriz da qualidade são estabelecidas a qualidade planejada e a qualidade projetada. Segundo AKAO (1996), existem dois pontos de vista para determinar a qualidade planejada: um é o ponto de vista do cliente, ou seja, quais são as qualidades mais importantes para ele, e o segundo é o da própria empresa, que compara ela própria com os concorrentes. A qualidade projetada deve ser considerada como o plano de melhoria para as características da qualidade do produto. Segundo Cheng et al. (1995), o plano de melhoria significa definir novos valores para as características da qualidade ou manter os valores atuais, com o objetivo de superar os concorrentes naqueles itens de maior importância segundo o mercado. A matriz da Qualidade é o cerne do QFD. Os requisitos do cliente podem ser convertidos em requisitos de recursos da função do produto de forma eficiente, usando a matriz de relações nessa matriz. Na análise de função, todo o processo de concepção pode ser controlado pelo QFD. Os projetistas podem mapear as necessidades do cliente e sua importância para as funções do produto e respectiva importância com a casa da qualidade do QFD (DU et al., 2013).

De acordo com Fernandes e Rebelato (2006), em cada ciclo de QFD relacionam-se as necessidades da qualidade (“o que se espera”) com os requisitos da qualidade ("como se pretende fazer"), identificando-se, na matriz de relações, a intensidade do relacionamento entre eles por meio de "símbolos de relações”. Cada símbolo tem um peso numérico representando esta intensidade. A importância relativa é uma classificação (priorização) de cada necessidade da qualidade (“o que se espera”). Essas necessidades são ponderadas segundo o grau de importância para o cliente, atribuindo-se a cada uma um valor numérico. Na parte superior da figura, identificam-se os relacionamentos entre os requisitos da qualidade (matriz de correlações). Estes relacionamentos identificam o grau de correlação entre os requisitos, auxiliando na priorização e identificação de "soluções de compromisso” entre eles. As especificações do projeto (“quanto”) representam uma quantificação de cada requisito da qualidade. Os requisitos priorizados são calculados pela multiplicação do peso atribuído ao símbolo de relações (“forte”, “moderado”, “fraco”) pelo grau de importância relativa (BERK; BERK, 1997). Para melhor visualização a Figura 2 ilustra estas considerações.

Figura 2 - Representação simplificada do método QFD.

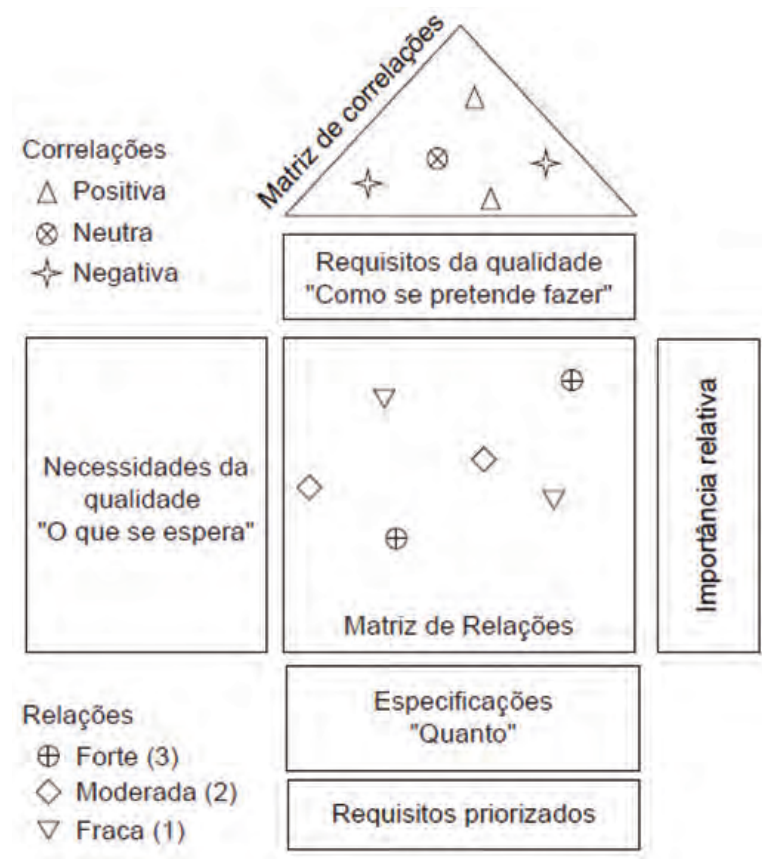

Fonte: Adaptado de Berk; Berk (1997, p. 205).

Conforme Peixoto e Carpinetti (1998), a força do QFD está em tornar tais relações (no sentido amplo, ou seja, a extração, a relação e a conversão) explícitas, permitindo a harmonização e priorização das várias decisões tomadas durante o processo de desenvolvimento do produto, bem como em potencializar o trabalho de equipe. Assim, o QFD é um método visual (utiliza-se principalmente de diagramas e matrizes), conectivo (os desdobramentos amarram as variáveis umas às outras) e priorizador (foca as atenções da equipe nas variáveis verdadeiramente importantes). De acordo com Cota Júnior e Cheng (2006), algumas das contribuições do QFD verificadas em um projeto aplicado em uma empresa de telecomunicações, como o auxílio na análise competitiva, a explicitação das relações de causa e efeito, e a indução de discussões e reflexões por parte da equipe, são comumente percebidas em projetos similares de aplicações do QFD para produtos tangíveis. Os benefícios advindos do QFD são em grande parte dependentes de fatores relacionados à cultura organizacional, tais como trabalho em equipe, quebra de barreiras interdepartamentais, consolidação de equipes multifuncionais, delegação de autoridade e responsabilidade para a tomada de decisões, entre outros (PEIXOTO; CARPINETTI, 1998). De acordo com Carnevalli et al. (2004), os benefícios da implantação do QFD são: 
- Fornece melhor visão sobre os concorrentes;

- Redução do tempo de lançamento de novos produtos;

- Redução do numero de alterações após lançamento;

- Melhoria da comunicação interfuncional;

- Redução de custos;

- Fortalecimento da pratica da Engenharia Simultânea;

- Melhoria do trabalho em grupo;

- Aumento da satisfação dos funcionários;

- Aumento da satisfação do cliente;

- Aumento da qualidade e confiabilidade.

Em questão de limitações, Poel (2007) descreve problemas metodológicos do QFD, entre eles a dificuldade de incorporar ao produto/processo demandas coletivas sem violar restrições de produção inicialmente estabelecidas. Carnevalli et al. (2004) também colocam as principais dificuldades para implementar o QFD:

- Dificuldade em função da estrutura organizacional da empresa;

- Falta de suporte gerencial;

- Comprometimento dos membros do grupo;

- Falta de recursos;

- Falta de experiência em QFD;

- Falta de tempo para conduzir a consulta aos clientes;

- Dificuldade em atribuir peso aos requisitos dos clientes;

- Dimensão das matrizes (grandes);

- Dificuldade em interpretar os requisitos dos clientes;

- Falta de treinamento no método;

- As matrizes não ajudam na sistematização;

- Conflitos de opiniões nos grupos.

Devido, ao se iniciar um estudo de QFD, haver normalmente um esforço consciente de delinear claramente quais são os objetivos a serem alcançados por aquele projeto específico de desenvolvimento de produto, o QFD pode ser considerado como um método que trata dos problemas de desenvolvimento de produto do tipo bem-definido ou bem-estruturado (CHENG, 2003). Os principais motivos que levam as empresas a iniciar a implantação do QFD são (LUCAS FILHO et al., 2010): (i) melhoria do processo de desenvolvimento de produtos; (ii) decisão a partir dos conhecimentos de suas vantagens e (iii) aumento da satisfação dos clientes. Ouvir os clientes é essencial, no entanto nem sempre é fácil traduzir para os produtos ou serviços os desejos dos consumidores, pois muitas vezes a linguagem utilizada não se adapta diretamente às especificações técnicas. O QFD contribui para vencer essas dificuldades, na realidade, o QFD auxilia na garantia da qualidade de processos, produtos e serviços (SONDA et al., 2000).

\section{Métodos adotados}

Primeiramente, cabe destacar que o tema QFD é abordado teórica e empiricamente na literatura. No entanto, quando se refere a investigação desse tema combinado especificamente com o desenvolvimento de produtos, considera-se não serem muitos os trabalhos encontrados no país. No entanto, esta afirmativa deve ser demonstrada. Nesse sentido, o presente trabalho tem como propósito realizar uma análise bibliográfica sobre o QFD, no contexto do desenvolvimento de novos produtos na produção nacional. O trabalho caracteriza-se como teórico conceitual, de análise da literatura, por meio da busca de artigos em bases de dados de periódicos no país, seguida pela organização e análise dessas publicações.

Em uma primeira fase, o trabalho buscou o fundamento para o desenvolvimento do trabalho, apresentado na seção anterior. Em seguida, foi feita a busca de artigos relacionados ao tema QFD e desenvolvimento de novos produtos tanto na principal base de dados de periódicos nacionais (Scielo - Scientific Eletronic Library Online) como também em revistas da engenharia de produção e da administração, que não constam nessa base. A Scielo é uma base de dados nacional de periódicos com uma coleção de 294 títulos periódicos brasileiros em diversas áreas do conhecimento. Para entrar nessa base, apenas são aceitos periódicos que atendam diversas exigências, tais como processo de avaliação, periodicidade, número mínimo de artigos publicados por ano, dentre outros fatores. Dentre alguns dos periódicos consultados nesta base de dados, destacam-se a Gestão \& Produção e a Produção, na área de engenharia de produção. Também está na base de dados a Revista Pesquisa Operacional, também ligada à engenharia de produção, mas considerada como fora de área em relação ao tema.

De forma intencional, para não limitar o levantamento e análise somente a uma única base de dados com dois periódicos da engenharia de produção, foram pesquisados periódicos específicos relacionados a área de engenharia de produção e administração. O Quadro 1 apresenta a lista de periódicos pesquisados e sua respectiva classificação no Qualis-CAPES das Engenharias III. 
Quadro 1 - Periódicos e Classificação no Qualis-CAPES de 2012 para a área de Engenharias III.

\begin{tabular}{|c|c|}
\hline Periódicos & $\begin{array}{l}\text { Qualis- } \\
\text { CAPES } \\
-2012\end{array}$ \\
\hline Gestão \& Produção & B2 \\
\hline Gestão da Produção, Operações e Sistemas (GEPROS) & B5 \\
\hline Pesquisa \& Desenvolvimento Engenharia de Produção & B5 \\
\hline Produto \& Produção (Impresso) & B4 \\
\hline Revista Produção Online & B4 \\
\hline Relatórios de Pesquisa em Engenharia de Produção (UFF) & B5 \\
\hline Revista Carioca de Produção & B5 \\
\hline Revista de Administração (FEA-USP) & B4 \\
\hline Revista de Administração (Belo Horizonte - Impresso) & B5 \\
\hline Revista de Administração Contemporânea (RAC - Impresso) & B4 \\
\hline Revista de Administração, Contabilidade e Economia (RACE) & B4 \\
\hline Revista de Administração e Inovação (RAI) & B4 \\
\hline Revista de Administração da UFSM & B4 \\
\hline Revista de Administração da UNIMEP & B5 \\
\hline Revista de Ciências da Administração (CAD/UFSC) & B5 \\
\hline Revista de Economia e Administração (Impresso) & B4 \\
\hline Revista de Estudos de Administração (UNIJUÍ) & B5 \\
\hline Revista Eletrônica de Administração (Garça - Online) & B5 \\
\hline Revista Eletrônica Produção \& Engenharia & B4 \\
\hline Revista Ingepro: Inovação, Gestão e Produção & B5 \\
\hline Revista Pensamento Contemporâneo em Administração (UFF) & B5 \\
\hline Revista Sistemas e Gestão & B5 \\
\hline
\end{tabular}

Fonte: Elaborado pelos autores.
Todas as fontes de pesquisa são nacionais, intencionalmente para manter o recorte nas publicações no país, havendo possibilidade de expandir a pesquisa futuramente. Ratifica-se que antes de se iniciar a revisão da literatura, necessitou-se o estabelecimento de palavras chaves que direcionariam a pesquisa nos periódicos selecionados e que poderiam posteriormente enquadrá-los de acordo com esta classificação. Para esta busca foram relacionadas as seguintes palavras-chave: QFD (com variantes Quality Function Deployment ou Desdobramento da Função Qualidade) e Desenvolvimento de Novos Produtos.

Uma vez conhecida e definida cada uma das palavras chaves usadas para a busca bibliográfica, os artigos foram recuperados, e a análise dos dados obtida por meio de leitura desses. Os dados de cada artigo foram transcritos para fichas de registro individual, para possibilitar a análise dos mesmos. De uma maneira geral, o trabalho foi executado conforme mostra a Figura 3.

\section{Resultados e discussão}

Por meio do conhecimento das principais definições para o desenvolvimento deste trabalho, possibilitou-se a identificação e análise das práticas do QFD existentes na literatura no país. Os resultados alcançados foram relativos a:

- Análise total de artigos sobre QFD, onde foi feita uma classificação, quantificação e estratificação geral dos artigos nos periódicos encontrados;

Figura 3 - Estrutura para desenvolvimento do trabalho.

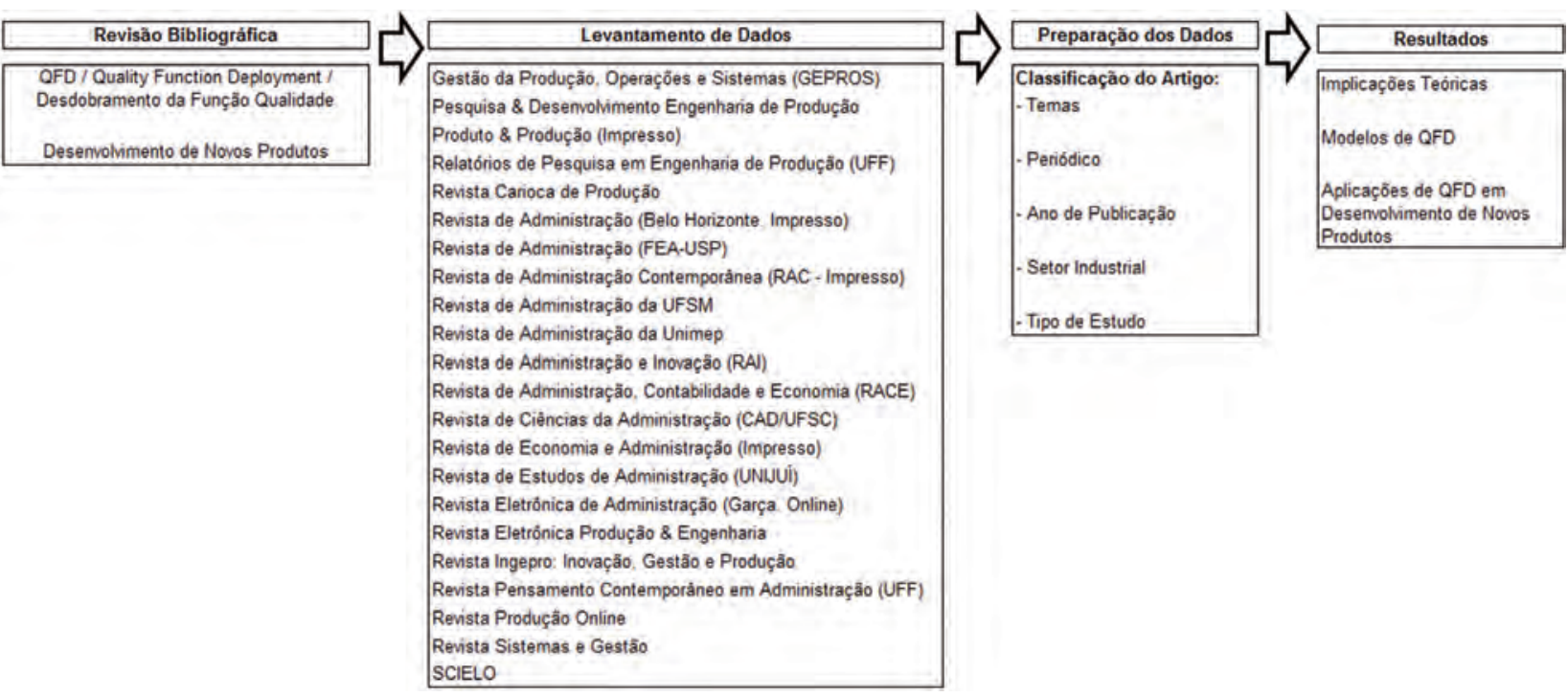

Fonte: Elaborada pelos autores. 
- Estratificação dos artigos sobre QFD associados ao desenvolvimento de novos produtos, permitindo a análise específica quanto aos periódicos identificados, bem como os anos das publicações e tipos de estudos;

- Práticas de desenvolvimento de novos produtos através do QFD de acordo com os setores organizacionais, identificando-se os principais setores que utilizam este método, bem como seus principais resultados com o método.

\subsection{Análise do total de artigos}

Após a definição do quadro teórico que norteou a pesquisa, selecionaram-se para participar do estudo 59 artigos sobre QFD. Conforme ilustrado na Figura 4, observa-se a predominância dos artigos identificados na base de dados Scielo (39\%), o que justifica a importância desse portal aberto de publicações em periódicos no
Brasil. A Revista Produção Online também apresentou um número expressivo de artigos (32\%). Diversos periódicos pesquisados não apresentaram artigos referentes ao QFD, sendo estes: Pesquisa \& Desenvolvimento Engenharia de Produção; Relatórios de Pesquisa em Engenharia de Produção (UFF); Revista Carioca de Produção; Revista de Administração (FEA-USP); Revista de Administração (Belo Horizonte - Impresso); Revista de Administração Contemporânea (RAC - Impresso); Revista de Administração, Contabilidade e Economia (RACE); Revista de Administração e Inovação (RAI); Revista de Administração da UFSM; Revista de Administração da UNIMEP; Revista de Ciências da Administração (CAD/ UFSC); Revista de Economia e Administração (Impresso); Revista de Estudos de Administração (UNIJUÍ); Revista Eletrônica de Administração (Garça - Online); Revista Eletrônica Produção \& Engenharia e Revista Pensamento Contemporâneo em Administração (UFF). Sendo assim, estes periódicos não estão sendo considerados na Figura 4.

Figura 4 - Percentual de artigos relacionados ao QFD por periódicos.
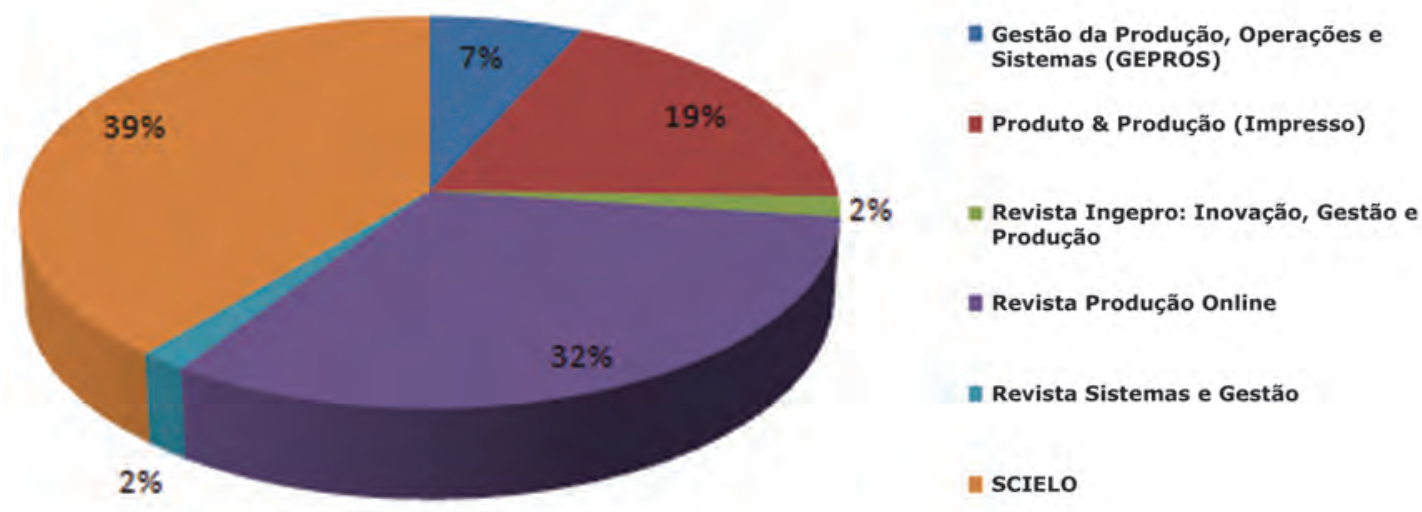

Fonte: Elaborada pelos autores.

Dos periódicos consultados na base de dados Scielo, 10 apresentaram artigos sobre o QFD, sendo estes: Caderno de Saúde Pública; Revista Acta Amazônica; Revista Ciência e Agrotecnologia; Revista Ciência e Tecnologia de Alimentos; Revista Gestão e Produção; Revista Horticultura Brasileira; Revista Latino Americana Enfermagem; Revista Polímeros; Revista Produção; Revista Scientia Agrícola.
Por meio da análise do Quadro 2, observa-se a concentração dos artigos relacionados ao desenvolvimento de produtos, correspondendo a quase metade $(42,4 \%)$ dos artigos encontrados (25 artigos). Esta concentração justifica-se pelo fato de as empresas buscarem melhorias no seu processo de desenvolvimento de novos produtos, e querer aumentar a satisfação de seus clientes. 
Quadro 2 - Tema e quantidade de artigos por título de periódico - Estratificado.

\begin{tabular}{|c|c|c|c|c|c|c|c|c|c|c|c|c|c|c|c|c|}
\hline \multirow[b]{2}{*}{ Periódicos } & \multicolumn{16}{|c|}{ Temas } \\
\hline & 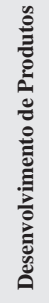 & 总 & 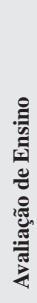 & 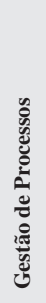 & 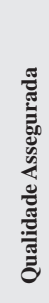 & 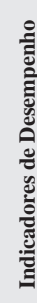 & 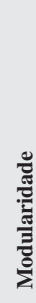 & 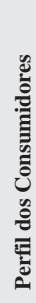 & 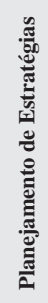 & 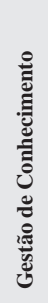 & 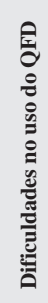 & 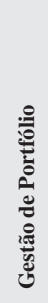 & 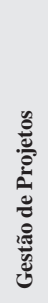 & 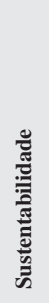 & 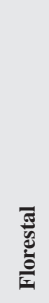 & बृّ \\
\hline Caderno de Saúde Pública & - & 1 & - & - & - & - & - & - & - & - & - & - & - & - & - & 1 \\
\hline Gestão da Produção, Operações e Sistemas (GEPROS) & 1 & 1 & 2 & - & - & - & - & - & - & - & - & - & - & - & - & 4 \\
\hline Produto \& Produção (Impresso) & 5 & 1 & - & 2 & - & 1 & 1 & - & - & 1 & - & - & - & - & - & 11 \\
\hline Revista Acta Amazônica & 1 & - & - & - & - & - & - & - & - & - & - & - & - & - & - & 1 \\
\hline Revista Ciência e Agrotecnologia & - & - & - & - & - & - & - & 1 & - & - & - & - & - & - & - & 1 \\
\hline Revista Ciência e Tecnologia de Alimentos & 1 & - & - & - & - & - & - & 1 & - & - & - & - & - & - & - & 2 \\
\hline Revista Gestão e Produção & 4 & - & 1 & - & 1 & - & - & - & 1 & - & - & - & - & - & - & 7 \\
\hline Revista Horticultura Brasileira & 1 & - & - & - & - & - & - & - & - & - & - & - & - & - & - & 1 \\
\hline Revista Ingepro: Inovação, Gestão e Produção & 1 & - & - & - & - & - & - & - & - & - & - & - & - & - & - & 1 \\
\hline Revista Latino Americana Enfermagem & - & 1 & - & - & - & - & - & - & - & - & - & - & - & - & - & 1 \\
\hline Revista Polímeros & 1 & - & - & - & - & - & - & - & - & - & - & - & - & - & - & 1 \\
\hline Revista Produção & 6 & - & - & - & - & - & - & - & - & - & 1 & - & - & - & - & 7 \\
\hline Revista Produção Online & 3 & 4 & 2 & 2 & 2 & 1 & 1 & - & 1 & - & - & 1 & 1 & 1 & - & 19 \\
\hline Revista Scientia Agrícola & - & - & - & - & - & - & - & - & - & - & - & - & - & - & 1 & 1 \\
\hline Revista Sistemas e Gestão & 1 & - & - & - & - & - & - & - & - & - & - & - & - & - & - & 1 \\
\hline Total & 25 & 8 & 5 & 4 & 3 & 2 & 2 & 2 & 2 & 1 & 1 & 1 & 1 & 1 & 1 & 59 \\
\hline
\end{tabular}

Fonte: Elaborado pelos autores.

Nota-se que dos periódicos supracitados no Quadro 2, somente 6 deles apresentaram artigos sobre QFD relacionados a mais de um dos 15 temas apresentados, ficando a Revista Produção Online com a maior diversificação, tendo publicados artigos pertinentes a 11 temas. Em segundo lugar vem a Revista Produto \& Produção apresentando artigos pertinentes a 6 temas, seguida pela Revista Gestão \& Produção, tendo artigos pertinentes a 5 temas. O restante dos periódicos apresentaram artigos concentrados em 3 temas ou menos.

\subsection{Estratificação dos artigos relacionados ao desenvolvimento de novos produtos}

Especificamente com relação a classificação aos artigos em desenvolvimento de novos produtos como tema de estudo (25 no total), estes foram encontrados especificamente nos seguintes periódicos (percentuais entre parênteses):
- Produção (24\%);

- Produto \& Produção (20\%);

- Gestão \& Produção (16\%);

- Revista Produção Online (12\%);

- Gestão da Produção, Operações e Sistemas - GEPROS (4\%);

- Acta Amazônica (4\%);

- Revista Ciência e Tecnologia de Alimentos (4\%);

- Revista Horticultura Brasileira (4\%);

- Revista Ingepro: Inovação, Gestão e Produção (4\%);

- Revista Polímeros (4\%);

- Gestão \& Sistemas (4\%).

O resultado com relação ao número de publicações de artigos por ano referente ao desenvolvimento de novos produtos é mostrado na Figura 5, onde consta uma média de pouco menos de duas publicações por ano, do período de 1998 até 2012. Ressalta-se que, nesse período, os anos 
Figura 5 - Evolução das publicações por ano.

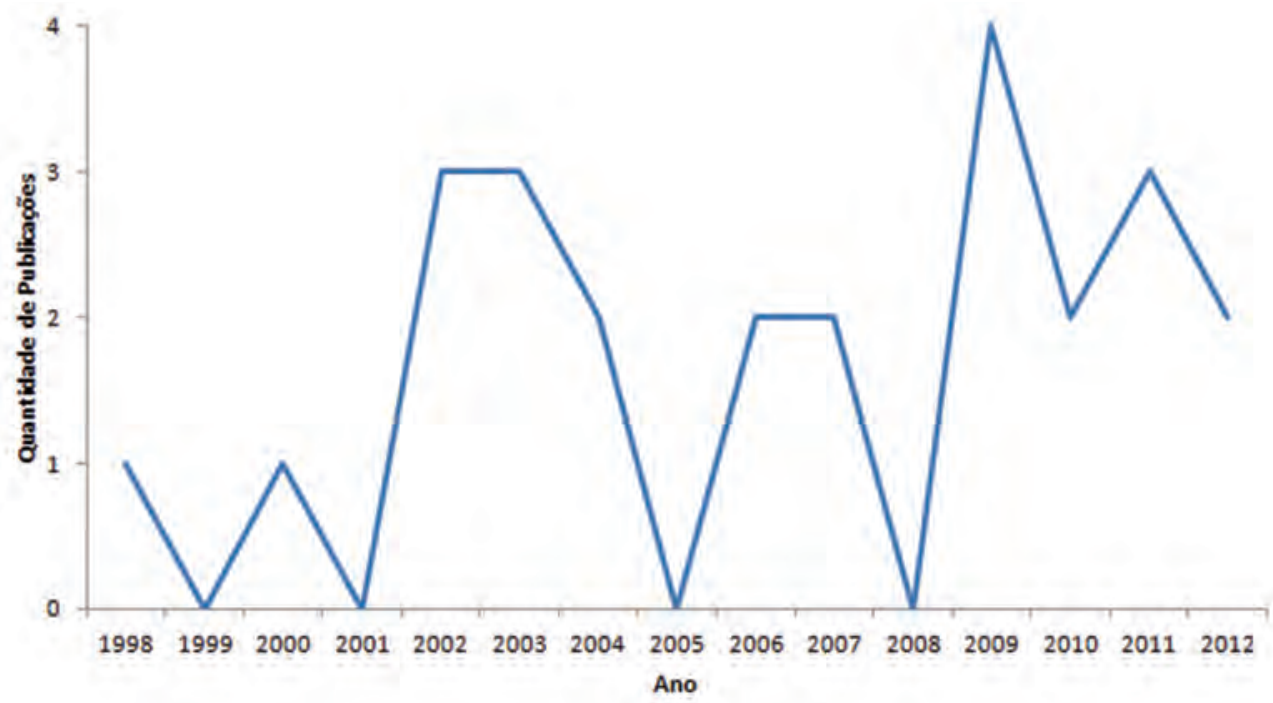

Fonte: Elaborada pelos autores.

\begin{tabular}{|c|c|c|c|c|c|c|c|c|c|c|c|c|c|c|c|c|}
\hline \multirow[b]{2}{*}{ Periódicos } & \multicolumn{16}{|c|}{ Anos } \\
\hline & $\stackrel{\infty}{\stackrel{\infty}{\sigma}}$ & ஓ् & ఫ్రి & $\overline{\stackrel{\sim}{~}}$ & ర్సి & 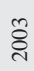 & ఫ্ণ & 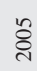 & \&્సి & ثे & $\stackrel{\sim}{N}_{0}^{\infty}$ & ષ્సి & $\stackrel{\circ}{\stackrel{\sim}{~}}$ & $\overrightarrow{\text { ते }}$ & $\underset{\tilde{N}}{\tilde{N}}$ & స్్ㅇ \\
\hline Gestão da Produção, Operações e Sistemas (GEPROS) & - & - & - & - & - & - & - & - & - & - & - & - & - & - & 1 & 1 \\
\hline Produto \& Produção (Impresso) & - & - & - & - & 1 & - & 1 & - & 1 & - & - & 3 & - & - & - & 6 \\
\hline Revista Acta Amazônica & - & - & - & - & - & - & - & - & - & - & - & - & 1 & - & - & 1 \\
\hline Revista Ciência e Tecnologia de Alimentos & - & - & - & - & - & 1 & - & - & - & - & - & - & 1 & - & - & 2 \\
\hline Revista Gestão e Produção & 1 & - & - & - & - & - & 1 & - & - & 1 & - & - & - & - & - & 3 \\
\hline Revista Horticultura Brasileira & - & - & - & - & 1 & - & - & - & - & - & - & - & - & - & - & 1 \\
\hline Revista Ingepro: Inovação, Gestão e Produção & - & - & - & - & - & - & - & - & - & - & - & - & - & 1 & - & 1 \\
\hline Revista Polímeros & - & - & - & - & - & - & - & - & - & - & - & - & - & - & - & - \\
\hline Revista Produção & - & - & 1 & - & 1 & - & - & - & - & 1 & - & 1 & - & 2 & - & 6 \\
\hline Revista Produção Online & - & - & - & - & - & 2 & - & - & - & - & - & - & - & - & 1 & 3 \\
\hline Revista Sistemas e Gestão & - & - & - & - & - & - & - & - & 1 & - & - & - & - & - & - & 1 \\
\hline Total & 1 & - & 1 & - & 3 & 3 & 2 & - & 2 & 2 & - & 4 & 2 & 3 & 2 & 25 \\
\hline
\end{tabular}

Fonte: Elaborado pelos autores.

de 1999, 2001, 2005 e 2008 não apresentaram publicações específicas sobre o tema nos periódicos analisados.

Nota-se que existe uma quantidade mais acentuada de publicações na literatura mais recente (a partir de 2009), conforme mostrado no quadro 3. No entanto, os trabalhos de implementação do desenvolvimento de novos produtos por meio do QFD são relativamente escassos, quando comparados com a literatura internacional, em função do pequeno número de artigos encontrados nas principais fontes de pesquisa nacionais da engenharia de produção e administração.

\subsection{Desenvolvimento de novos produtos através do QFD de acordo com os setores organizacionais e tipos de estudos}

Com o intuito de apresentar o desenvolvimento de novos produtos por meio do QFD utilizado por organizações em geral, a Figura 6 objetiva apresenta os setores industriais em que os artigos encontrados se enquadram, identificando assim certa diversificação de setores econômicos. 
Figura 6 - Quantidade de publicações por setor industrial.

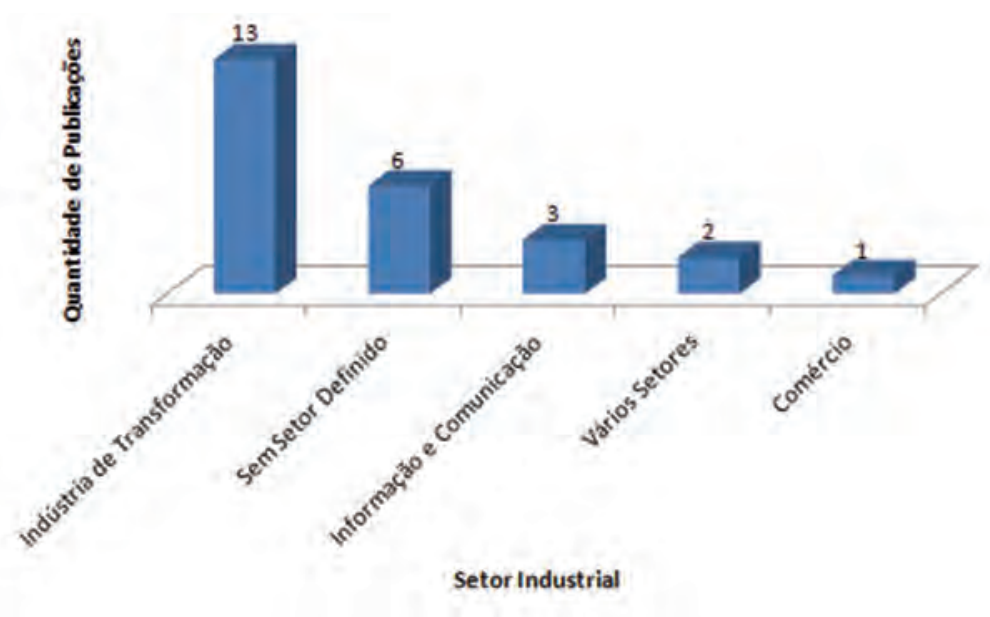

Fonte: Elaborada pelos autores.

Por meio da figura 6 observa-se a maior parte dos estudos relacionados à indústria de transformação (52\%), destacando-se entre estes estudos a fabricação de móveis, de materiais plásticos e de produtos alimentícios. Em seguida apresentam-se, com significativo percentual (24\%), estudos sem definição de setores industriais. Os demais 24\% dividem-se em estudos relacionados aos setores de informação e comunicação (12\%), vários setores (8\%), sendo estudos envolvendo simultaneamente: indústria de transformação, educação e serviços; e, indústria de transformação, água, eletricidade e gás; e comércio (2\%).

Por meio de análises efetuadas nos artigos específicos de desenvolvimento de novos produtos por meio do QFD, extraíram-se os resultados principais, os quais são apresentados no quadro 4.

Quadro 4 - Principais resultados dos artigos por setor industrial.

\begin{tabular}{|l|l|}
\hline \multicolumn{1}{|c|}{ Setor Industrial } & \multicolumn{1}{c|}{ Principais Resultados dos Artigos } \\
\hline Indústria de Transformação & $\begin{array}{l}\text { Desenvolvimento de produtos mais sustentáveis; melhorias nas habilidades de planejamento; melhoria de comunicação entre } \\
\text { as áreas; avaliação do alinhamento estratégico, qualidade e rentabilidade de projetos de produtos; Identificação e tradução das } \\
\text { demandas de qualidade; melhorias relacionadas as demandas priorizadas. }\end{array}$ \\
\hline Vários Setores & $\begin{array}{l}\text { Identificação de dificuldades e benefícios de forma comparativa do QFD; aumento da satisfação dos clientes; diagnóstico } \\
\text { geral da literatura sobre o QFD; apresentação de um guia para a intervenção no sistema de desenvolvimento de produtos nas } \\
\text { empresas. }\end{array}$ \\
\hline Sem Setor Definido & $\begin{array}{l}\text { Garantia de conexão entre decisões tomadas durante o processo de desenvolvimento de produto; determinação das prioridades } \\
\text { através da conversão de pesos; redução de custos e frequência de manutenção. }\end{array}$ \\
\hline Informação e Comunicação & $\begin{array}{l}\text { Percepção da necessidade da definição antecipada das características de um sistema para o desenvolvimento de um software; } \\
\text { determinação dos pontos a serem reformulados em um produto; geração de subsídios para a incorporação de uma proposta de } \\
\text { reformulação com características antes ignoradas. }\end{array}$ \\
\hline Comércio & Adaptação de produtos para mercados diferenciados a partir da engenharia reversa, utilizando-se das matrizes do QFD. \\
\hline
\end{tabular}

Fonte: Elaborado pelos autores.

A maioria dos artigos pesquisados apresenta esboços das construções das matrizes do QFD, mostrando os métodos de desenvolvimentos e a estruturação pelas quais os resultados ocorreram. Nota-se que muitos resultados diferenciados são alcançados através da utilização do QFD para o desenvolvimento de novos produtos, mas observa-se que um dos resultados que mais é comentado refere-se à aproximação que o QFD traz as equipes que trabalham utilizando o mesmo, o que faz com que as empresas tornem-se mais ágeis em termos de comunicação, e, consequentemente, em dar respostas aos solicitantes. Ao se aprofundar nos resultados, nota-se que os mesmos são voltados tanto internamente, no sentido de se planejar ações e decisões, estreitar relacionamentos intersetoriais, reduzir custos, eliminar desperdícios, quanto externamente, no sentido de entender o direcionamento do mercado, desenvolver produtos sustentáveis, aumentar a satisfação dos clientes, dentre outros. Nota-se pelas situações relatadas nas publicações, que as organizações após fazerem uso do QFD em algumas frentes de trabalho, passam a adotá-lo como um método de gestão para diversas outras situações, devido sua abrangência e eficácia nos resultados.

Em relação as abordagens metodológicas de pesquisa adotadas na publicações, a Figura 7 apresenta os tipos de abordagens resultantes da análise dos artigos. 
Figura 7 - Quantidade de publicações por tipo de estudo.

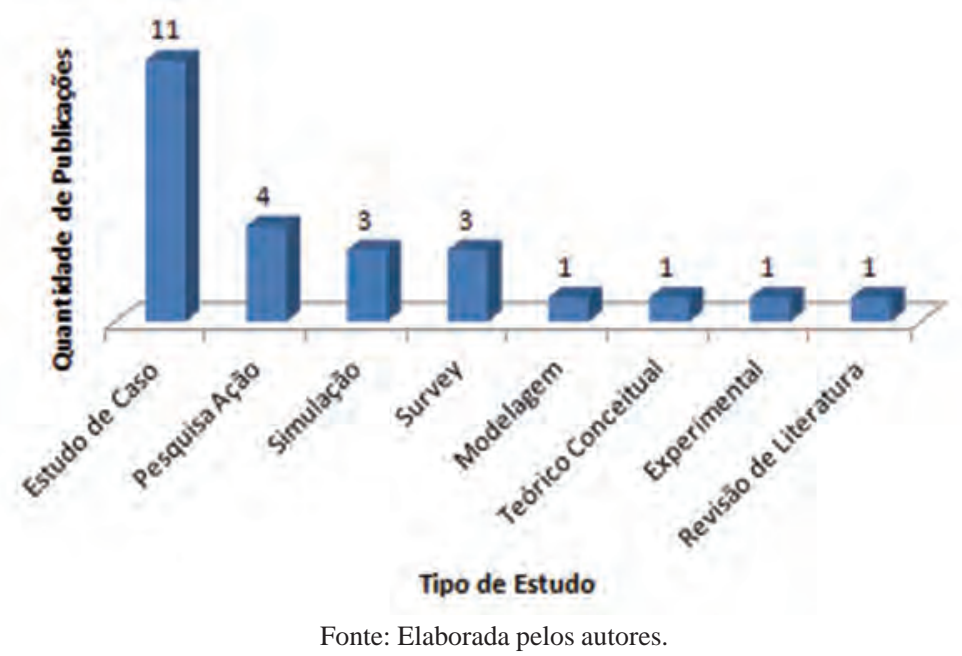

Conforme é mostrado na Figura 7, existe uma predominância de artigos desenvolvidos por meio do estudo de caso (44\%), seguido pela pesquisa-ação (16\%). Os trabalhos desenvolvidos por meio de simulação e survey corresponderam a $12 \%$ para cada um dos tipos. A modelagem, estudo teórico conceitual, trabalho experimental e revisão de literatura apresentaram-se com $4 \%$ para cada um destes tipos de estudos.

De acordo com Yin (2001), da essência de um estudo de caso, a principal tendência em todos os tipos de estudo de caso, é que ela tenta esclarecer uma decisão ou um conjunto de decisões: o motivo pelo qual foram tomadas, como foram implementadas e com quais resultados. Os estudos de casos encontrados na busca bibliográfica mostraram-se bastante diversificados, por terem apresentado análises de fenômenos por meio de diversas fontes de evidência, tais como: entrevistas, análise documental, dentre outras. Notou-se que alguns estudos se aprofundaram mais na coleta e análise de dados, enquanto outros trataram destas fases do estudo de caso de forma mais sucinta.

Em síntese, os resultados deste estudo teórico conceitual mostram que:

- Do total de periódicos pesquisados, 15 apresentaram artigos referentes ao QFD, sendo 10 destes periódicos da base Scielo;

- Dos 59 artigos sobre QFD encontrados, 42,4\% referem-se ao desenvolvimento de novos produtos, sendo que estes foram identificados em 11 periódicos diferentes, portanto, bastante diversificado;

- Além do tema desenvolvimento de novos produtos, mais 14 temas conjuntos com o QFD foram listados;

- Foi constatada uma média de pouco menos de duas publicações por ano sobre o desenvolvimento de novos produtos por meio do QFD;
- O setor industrial relacionado à indústria de transformação apresentou a mais da metade (52\%) dos artigos referentes ao desenvolvimento de novos produtos por meio do QFD;

- O tipo de estudo que predominou dentre os artigos referentes ao desenvolvimento de novos produtos através do QFD foi o estudo de caso (44\%).

Todos esses resultados possibilitam desenvolver um maior entendimento sobre o tema QFD voltado para o desenvolvimento de novos produtos, possibilitando a comparação dos resultados das publicações realizadas nos diversos setores industriais.

\section{Considerações finais}

Este trabalho abordou a identificação e análise da literatura existente em periódicos no Brasil no tema QFD, abordando de forma mais ampla as publicações sobre o tema. Ao detalhar mais a análise, constata-se que artigos com maior quantidade relacionam-se com o desenvolvimento de novos produtos. Dentre os vários temas encontrados nos artigos sobre QFD, nota-se a predominância de artigos sobre este tema, o que mostra que este é um tema específico mais abordado. Apesar disto, considera-se que a prática do desenvolvimento de novos produtos por meio do QFD, a partir das publicações no país, não é tão amplamente explorada, em função da baixa quantidade de artigos encontrados nos periódicos analisados. Com relação aos setores que utilizam o QFD para o desenvolvimento de novos produtos, constata-se a predominância dos setores ligados à indústria de transformação. Também nota-se uma concentração de publicações referentes ao tema supracitado em poucos periódicos. A partir da síntese anterior, conclui-se que o tema (QFD) é razoavelmente consolidado no país, mas que existem oportunidades de melhorias não cobertas por parte das publicações vigentes. Estas análises das publicações sobre o desenvolvimento de novos produtos através do QFD no Brasil, desenvolvidas 
até o momento, podem ser consideradas de grande valia para que se possa dar continuidade ao estudo nesse tema, efetuando análises das publicações internacionais.

\section{Agradecimentos}

Os autores agradecem ao apoio do CNPq para a realização do presente trabalho e aos revisores da Produto \& Produção pelas recomendações realizadas visando a melhoria do presente trabalho.

\section{Referências}

AKAO, Y. Introdução ao desdobramento da qualidade. Belo Horizonte: Fundação Christiano Ottoni, 1996.

AKAO, Y. Quality Function Deployment: integrating customer requirements into product design. Cambridge: Productivity Press, 1990.

ANZANELLO, M.J.; LEMOS, F.O.; ECHEVESTE, M.E. HENG, L.C. Aprimorando Produtos Orientados ao Consumidor Utilizando Desdobramento da Função Qualidade (QFD) e Previsão de Demanda. Produto \& Produção, v. 10, n. 2, p. 1-27, 2009.

BERK, J; BERK, S. Administração da qualidade total: o aperfeiçoamento contínuo. São Paulo: IBRASA, 1997.

BOUCHEREAU, V.; ROWLANDS, H. Methods and techniques to help quality function deployment (QFD). Benchmarking: An International Journal, v. 7, n. 1, p. 8- 19, 2000.

BURKE, E.; KLOEBER JR, J.M.; DECKRO; R.F. Using and abusing QFD scores. Quality Engineering, v. 15, n. 1, p. 9-21, 2002.

BÜYÜKÖZKAN, G.; ÇIFÇI, G. An integrated QFD framework with multiple formatted and incomplete preferences: A sustainable supply chain application. Applied Soft Computing, v. 13, n. 9, p. 3931-3941, 2013.

CARNEVALLI, J.A.; SASSI, A.C. e CAUCHICK MIGUEL, P.A. Aplicação do QFD no desenvolvimento de produtos: levantamento sobre seu uso e perspectivas para pesquisas futuras. Gestão e Produção, v. 11, n. 1, p. 33-49, 2004.

CAUCHICK MIGUEL, P. A., CARNEVALLI, J. A. Aplicações não-convencionais do desdobramento da função qualidade. São Paulo: Artliber, 2006.
CAUCHICK MIGUEL, P.A.; TELFSER, M.; MARUCA, A.; GALLONETTI, A.; SARACURA, A.; MARTINS, L.; HORI, M.; RIBEIRO, P.; CAMPOS, R.; MARCONATO, T.; MORA, V. Desdobramento da Qualidade no Desenvolvimento de Filmes Flexíveis para Embalagens. Polímeros: Ciência e Tecnologia, v. 13, n. 2, p. 87-94, 2003.

CHENG, L. C. QFD em desenvolvimento de produto: características metodológicas e um guia para intervenção. Revista Produção On Line, v. 3, n. 2, 2003.

CHENG, L.C. et al. QFD: Planejamento da qualidade. Belo Horizonte: Fundação Christiano Ottoni, 1995.

CHENG, L.C.; MELOFILHO, L.D.R. QFD-Desdobramento da Função Qualidade na Gestão de Desenvolvimento de Produtos. São Paulo: Editora Blücher, 2007.

COTA JÚNIOR, M. B. G.; CHENG, L. C. Aplicação do QFD e do PCP a produtos digitais em uma empresa brasileira de telecomunicações móveis. Revista Eletrônica Sistemas e Gestão, v. 1, n. 3, p. 258-275, 2006.

DU, Y. et al. Reuse-oriented redesign method of used products based on axiomatic design theory and QFD. Journal of Cleaner Production, v. 39, p. 79-86, 2013.

FERNANDES, J.M.R.; REBELATO, M.G. Proposta de um método para integração entre QFD e FMEA. Gestão \& Produção, v. 13, n. 2, p. 245-259, 2006.

FORMAGGIO, I.A.; CAUCHICK MIGUEL, P.A. Múltiplo Estudo de Casos sobre a Inserção do QFD no Processo de Desenvolvimento de Novos Produtos. Produto \& Produção, v. 10, n. 2, p. 62-86, 2009.

GOVERS, C.P.M. What and how about quality function deployment (QFD). International Journal of Production Economics, v. 46-47, n. 1, p. 575-585, 1996.

KING, R. Listening to the Voice of the Customer: Using the Quality Function Deployment System. National Productivity Review, New York, v. 6, n. 3, p. 277-281, 1987.

LUCAS FILHO, F.C.; PIO, N.S.; FERREIRA, D.R. Método QFD como Ferramenta para Desenvolvimento Conceitual de Produtos de Madeiras da Amazônia. Acta Amazonia, v. 40, n. 4, p. 675-686, 2010.

NAHM, Y. A novel approach to prioritize customer requirements in QFD based on customer satisfaction function for customer-oriented product design. Journal of Mechanical Science and Technology, v. 27, n. 12, p. 3765-3777, 2013. 
PEIXOTO, M.O.C.; CARPINETTI, L.C.R. Aplicação de QFD integrando o modelo de Akao e o modelo QFD estendido. Gestão \& Produção, v. 5, n. 3, p. 221-238, 1998.

POEL, I. Methodological problems in QFD and directions for future development. Research in Engineering Design, v. 18, n. 1, p. 21-36, 2007.

RIBEIRO, J.L.D.; CUNHA, M.G.C.; ECHEVESTE, M.E. Desdobramento da qualidade: um plano de melhorias para retenção de clientes em clubes sociais e esportivo. ENEGEP. 21, 2001. Anais... . Salvador, 2001.
SONDA, F.A.; RIBEIRO, J.L.D.; ECHEVESTE, M.E. A aplicação do QFD no desenvolvimento de software: um estudo de caso. Produção, v. 10, n. 1, p. 51-75, 2000.

YIN, R.K. Estudo de Caso - Planejamento e Método. $2^{\mathrm{a}}$. Edição. São Paulo: Bookman, 2001.

Recebido em 14/10/2012. Aceito em 28/07/2014. 


\section{Apêndice 1 - Lista Completa de Artigos Identificados na Busca Bibliográfica (inclui os artigos citados e os que não foram citados no texto).}

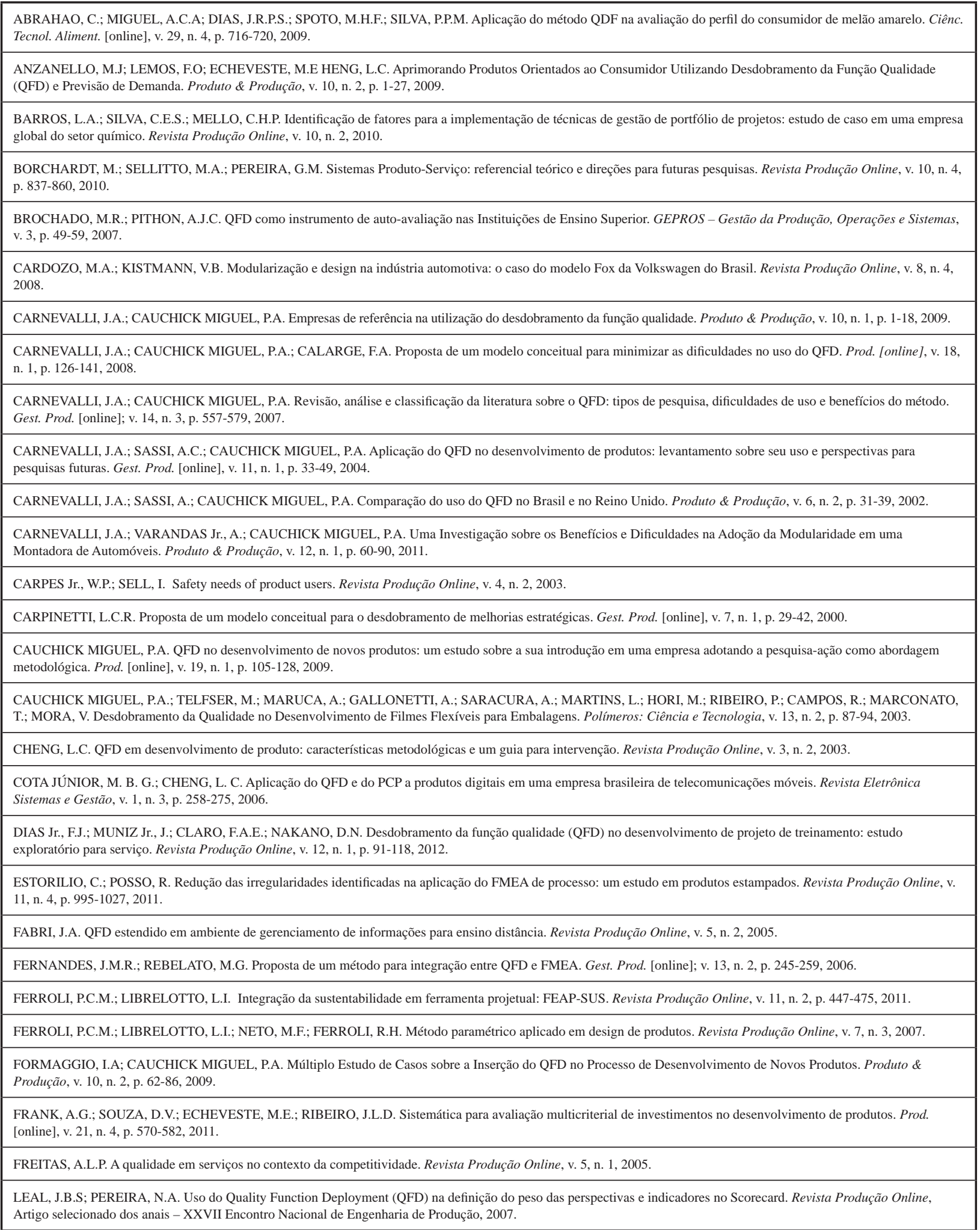


LUCAS FILHO, F.C.; PIO, N.S.; FERREIRA, D.R. Método QFD como Ferramenta para Desenvolvimento Conceitual de Produtos de Madeiras da Amazônia. Acta Amaz. [online], v. 40, n. 4, p. 675-686, 2010.

MAGNANO, P.F.; ECHEVESTE, M.E. Caracterização de um modelo mínimo para o PDS por meio de uma revisão sistemática de literatura. Produto \& Produção, v. 13, n. 1, p. 01-15, 2012.

MARCOS, S.K.; JORGE, J. T. Desenvolvimento de tomate de mesa, com o uso do método QFD (Desdobramento da Função Qualidade), comercializado em um supermercado. Hortic. Bras. [online], v. 20, n. 3, p. 490-496, 2002.

MARQUES, C.A.N.; RODRIGUES, M.F.C.; SUZUKI, J.A.; FARIA, A.F. Modelo de estudo de viabilidade aplicado ao processo de desenvolvimento de novos produtos. Inovação, Gestão e Produção, v. 3, n. 3, 2011.

MARX, A.M.; ECHEVESTE, M.E.S.; PAULA, I.C. Desdobramento da função qualidade aplicado ao projeto de um detergente sustentável. Prod. [online], v. 21, n. 4, p. 724$741,2011$.

MATEI, A.P.; ECHEVESTE, M.E.; CATEN, C.S.; ZOUAIN, R.N.A. Avaliação da qualidade demandada e diretrizes de melhoria no processo de interação UniversidadeEmpresa. Prod. [online], v. 22, n. 1, p. 27-42, 2012.

MATSUDA, L.M.; EVORA, Y.D.M.; BOAN, F.S. O método desdobramento da função qualidade - QFD - no planejamento do serviço de enfermagem. Rev. Latino-Am. Enfermagem [online], v. 8, n. 5, p. 97-105, 2000.

MELO FILHO, L.D.R.; CHENG, L.C. QFD na garantia da qualidade do produto durante seu desenvolvimento: caso em uma empresa de materiais. Prod. [online], v. 17, n. 3, p. 604-624, 2007.

MIGUEL, A.C.A.; SPOTO, M.H.F.; ABRAHAO, C.; SILVA, P.P.M. Aplicação do método QFD na avaliação do perfil do consumidor de abacaxi 'Pérola’. Ciênc. agrotec. [online], v. 31, n. 2, p. 563-569, 2007.

MILAN, M.; BARROS, J. W. D.; GAVA, J. L. Planning soil tillage using quality function deployment (QFD). Sci. agric. (Piracicaba, Braz.) [online], v. 60, n. 2, p. $217-221$, 2003.

MORAES, C.C.; CAUCHICK MIGUEL, P.A. Proposta de melhoria de um curso de mestrado em engenharia de produção por meio do uso do QFD - contexto de uma avaliação institucional. GEPROS - Gestão da Produção, Operações e Sistemas, v. 4, p. 23-36, 2007.

MURY, L.G.M.; FOGLIATTO, F.S. Adaptação de produtos para mercados diferenciados a partir da engenharia reversa. Prod. [online], v. 11, n. 2, p. 5-22, 2001. PEIXOTO, M.O.C.; CARPINETTI, L.C.R. Aplicação de QFD integrando o modelo de akao e o modelo QFD estendido. Gest. Prod. [online], v. 5, n. 3, p. 221-238, 1998.

PINNA, C.C.A.; CARVALHO, M.M. Gestão de escopo em projetos de aplicações web. Revista Produção Online, v. 8, n. 1, 2004.

PINTO, A.L.D.; PAIVA, C.L. Desenvolvimento de uma massa funcional pronta para tortas utilizando o método de Desdobramento da Função Qualidade (QFD). Ciênc. Tecnol. Aliment. [online], v. 30, supl.1, p. 36-43, 2010.

PINTO, S.H.B.; CARVALHO, M.M.; HOO, L.L. Programa seis sigma: aspectos sinérgicos com outras abordagens de gerenciamento da qualidade. Revista Produção Online, v. 9, n. 1, 2009.

PIOVEZAN, L.H. LAURINDO, F.J.B.; CARVALHO, M.M. Proposta de método para a formulação de estratégia em pequenas e médias empresas. Revista Produção Online, v. 8, n. 2, 2008.

PIZZOLATO, M.; TEN CATEN, C.S.; FOGLIATTO, F.S. Definição do prazo de garantia de um produto otimizado experimentalmente. Gest. Prod. [online], v. 12, n. 2, p. 239-253, 2005.

POSSO, R.; ESTORILIO, C. Identificação dos Fatores de Influência na Aplicação do Método Failure Mode and Effect Analysis- FMEA de Processo: um estudo em produtos estampados. Produto \& Produção, v. 10, n. 2, p. 87-107, 2009.

RODRIGUES, J.T.M.C; WERNER, L. Seis Sigma: características do programa em onze empresas situadas na região metropolitana de Porto Alegre. Revista Produção Online, v. 12, n. 1, p. 2-22, 2012.

ROYER, R.; FOGLIATTO, F.S. Indicador de viabilidade de customização em massa de produtos industriais. Produto \& Produção, v. 7, n. 1, p. 3-18, 2004.

ROZENFELD, H.; SILVA, S.L.; AMARAL, D.C.; ZANCUL, E.S. Um ambiente de interação universidade/empresa: experiências com a prática do desenvolvimento de produtos em uma fábrica integrada modelo. Produto \& Produção, v. 7, n. 1, p. 19-35, 2004

SANTOS, L.C.; GOHR, C.F.; VARVAKIS, G. Prioridades competitivas para a estratégia de operações de serviços: uma análise dos critérios de valor percebido de uma academia de ginástica. Revista Produção Online, v. 12, n. 1, p. 133-158, 2012.

SAVIOTTI, U. A Utilização dos Métodos QFD e FMEA para o Desenvolvimento de um Novo Produto. GEPROS - Gestão da Produção, Operações e Sistemas, n. 1, 2012.

SILVA, C.E.S.; GONÇALVES, T.C.C. Proposta de utilização do Quality Function Deployment (QFD) no sistema de análise de pontos críticos de controle (APPCC) na produção de refeições. GEPROS - Gestão da Produção, Operações e Sistemas, n. 3, p. 113-123, 2006

SILVA, S.L. A gestão do conhecimento e o desenvolvimento criativo de novos produtos: análise de um projeto selecionado pelo prêmio FINEP de inovação. Produto \& Produção, v. 8, n. 3, p. 81-92, 2005.

SILVEIRA, E.F.; WERNER, L. Proposta de método de priorização de processos a serem monitorados no controle estatístico de processo: uma aplicação em trocador de calor. Revista Produção Online, v. 11, n. 1, p. 116-135, 2011

SONDA, F.A.; RIBEIRO, J.L.D.; ECHEVESTE, M.E. A aplicação do QFD no desenvolvimento de software: um estudo de caso. Prod. [online], v. 10, n. 1, p. 51-75, 2000.

UNTERLEIDER, C.E.A.; SELLITTO, M.A. Aplicação do QFD em etapas iniciais do desenvolvimento de um odorizador de automóveis. Revista Produção Online, v. 12, n. 2, p. 377-401, 2012.

VIDAL, O.C.; KAMINSKI, P.C.; NETTO, S.N. Exemplos de aplicação do conceito de fábrica digital no planejamento de instalações para armação de carroçarias na indústria automobilística brasileira. Produto \& Produção, v. 10, n. 1, p. 75-84, 2009.

VOLPATO, L.F.; MENEGHIM, M.C.; PEREIRA, A.C.; AMBROSANO, G.M.B. Planejamento da qualidade nas unidades de saúde da família, utilizando o Desdobramento da

Função Qualidade (QFD). Cad. Saúde Pública [online], v. 26, n. 8, p. 1561-1572, 2010. 